\title{
A Tribute to Hans Grauert
}

\author{
Alan Huckleberry and Thomas Peternell, coordinating editors
}

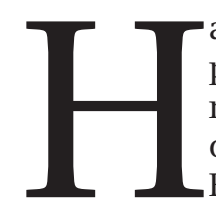

ans Grauert, one of the most creative, prolific, and scientifically influential mathematicians in the second half of the last century, was born on February 8, 1930, in Haren, a small town in northern Germany near the Dutch border. In the summer of 1949, he enrolled in the University of Mainz. One semester later he changed to Münster, where he soon met his lifelong friend and collaborator Reinhold Remmert. Heinrich Behnke, who had already built an internationally recognized research group in complex analysis in the 1930s, was the leading figure in Münster at this time. In particular, due to his connections to Henri Cartan and Heinz Hopf, after the war Behnke was able to keep Münster on the mathematical map. Karl Stein was already a well-established professor in the group and Friedrich Hirzebruch was a rising star. Grauert had entered, probably by chance, one of the most stimulating mathematical atmospheres in postwar Germany.

Profiting from the Münster complex analysis climate and an extended visit to Zürich at the invitation of Beno Eckmann, Grauert received his Ph.D. in Münster in 1954. His dissertation opened new connections between Kähler geometry and Stein theory. At the age of twenty-seven he obtained his habilitation with a series of papers on what is now known as the Oka-Grauert principle.

After spending the academic year 1957/1958 in Princeton at the Institute for Advanced Study and a semester at the IHES in Paris, in 1959 Grauert was named to the Gauss chair in Göttingen and became the successor of C. L. Siegel. Despite receiving offers from various distinguished universities, he remained in Göttingen for his entire academic life. Among numerous honors, he was an invited

Alan Huckleberry is professor of mathematics at the Fakultät für Mathematik, Ruhr Universität Bochum, Germany, and School of Engineering and Science, Jacobs University in Bremen, Germany. His email address is ahuck@cp 1x. rub.de.

Thomas Peternell is professor of mathematics at the Fakultät für Mathematik, Physik, Informatik, Universität Bayreuth, Germany. His email address is thomas . peterne11@ uni-bayreuth. de.

DOI: http://dx.doi.org/10.1090/noti1103

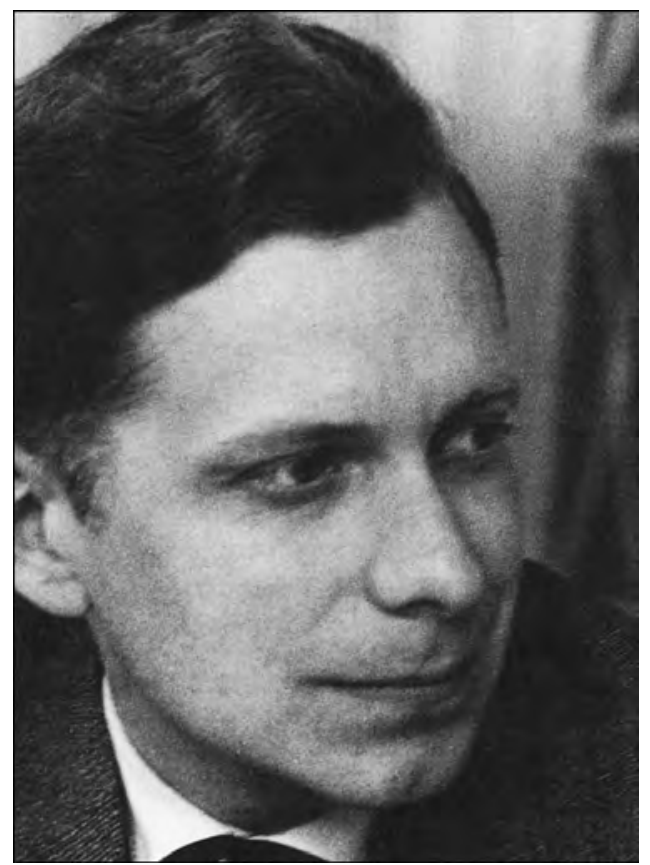

Hans Grauert, 1960.

speaker at the ICM in 1958, 1962 (plenary lecture), and 1966.

Grauert's contributions to mathematics incorporate all aspects of higher-dimensional complex analysis. Apart from his remarkable habilitation thesis where he proved the equivalence of the topological and holomorphic categories of various types of fiber bundles over Stein spaces, his works include

- foundational results on complex spaces and Stein theory (with Remmert),

- the direct image theorem and consequences in moduli theory,

- the solution to the Levi problem in the smooth case,

- pseudoconvex spaces and their applications in analytic and algebraic geometry,

- deformation theory, e.g., existence of versal deformations,

- vector bundles on projective spaces. 


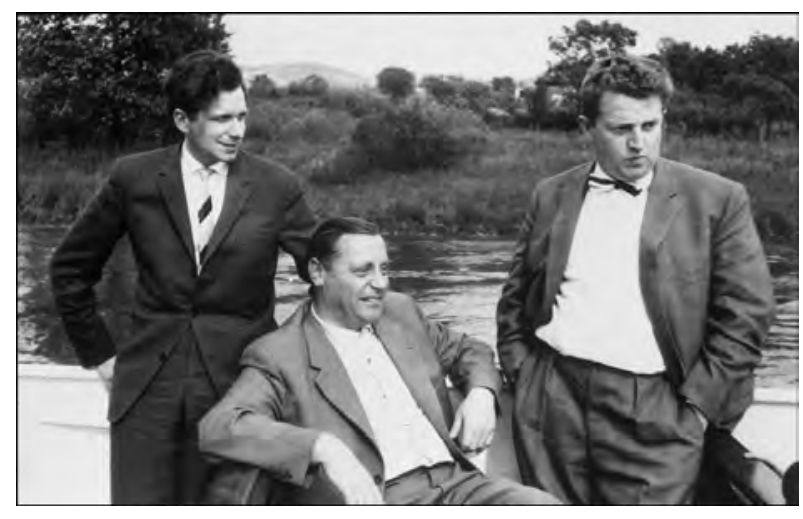

Grauert, Stein, and Remmert, 1957. Three men in a boat at the Arbeitstagung.

Three fundamental research monographs were jointly published with Remmert, and with various coauthors he published textbooks in the areas of real and complex analysis.

Grauert was an extremely successful "Akademischer Lehrer" in the classical German sense. In particular, he guided more than forty Ph.D. students, many of whom have continued the lines of research of their mentor. Two of them, Ingo Lieb and Günter Trautmann, together with Daniel Barlet, Jean-Pierre Demailly, Takeo Ohsawa, and Yum-Tong Siu, have contributed to this homage to Hans Grauert.

After a long illness, Hans Grauert passed away in September of 2011 at the age of 81 . His mathematical influence will last forever.

\section{Yum-Tong Siu}

\section{Hans Grauert in Memoriam}

The very first time I was introduced to the name and work of Professor Hans Grauert was at the seminar of Professor Solomon Bochner at Princeton. At the beginning of my first year at Princeton as a graduate student, I participated in Bochner's seminar, in which newcomers were assigned some papers to read and present. My assignment was Grauert's 1955 Mathematische Annalen paper on the characterization of holomorphically complete complex spaces. It was my first experience presenting a paper at such a seminar. I spent a great deal of time reading the paper and reorganizing it in the standard format of numbered definitions, lemmas, propositions, theorems, and corollaries in what I considered to be a better logical order of interdependence.

Yum-Tong Siu is professor of mathematics at Harvard University. His email address is siu@math. harvard. edu.
Only a few minutes into my presentation, Bochner stopped me and said that the way I presented it was definitely not how Grauert originally organized his paper. He went on to point out that Grauert was a real master and a master's own presentation contains the very valuable information of the master's thought process, which would be lost in a reorganization of the paper's contents. He illustrated his point by saying that when you play the music of a master composer, you are not supposed to substitute for it a variation of your own.

Needless to say, given all the effort and time I put into reorganizing Grauert's paper, I was very disappointed by Bochner's reaction. On the other hand, Bochner's comments served as a useful guide in my learning process from then on. While working on my Ph.D. thesis under the guidance of Professor Robert C. Gunning at Princeton, I spent a great deal of time poring over the original papers of Hans Grauert and avoided simply reading their expositions by other mathematicians. For me and the entire generation of mathematicians in several complex variables, Grauert's results plus all the explicit and implicit ideas in Grauert's papers are goldmines to explore and work on.

In the late 1950s and early 1960s, Grauert launched the new and richest phase of the theory of several complex variables on three fronts: (i) the Levi problem, (ii) the Oka principle for fiber bundles, and (iii) the direct image theorem.

For the Levi problem Grauert introduced his exceedingly ingenious bumping technique to construct global holomorphic functions. How to construct global holomorphic functions on abstractly defined complex manifolds and spaces is one of the most fundamental problems in complex geometry. Grauert's solution of the Levi problem reduces the construction of global holomorphic functions to the existence of strictly plurisubharmonic exhaustion functions. Prior to Grauert's solution of the Levi problem, the only abstractly defined complex manifolds for which a general method of constructing global holomorphic functions is available are noncompact Riemann surfaces. It is the method of minimizing Dirichlet integrals on Riemann surfaces, which is motivated by electrostatic potentials. Grauert's bumping technique of constructing holomorphic functions was a breakthrough of fundamental importance. Currently, besides Grauert's bumping technique, for construction of holomorphic functions there is also available the technique of $L^{2}$ estimates of $\bar{\partial}$ due to Morrey, Andreotti-Vesentini, Kohn, Hörmander, and others. However, for situations of singular spaces and sections of coherent sheaves, Grauert's method remains the only directly applicable tool. 


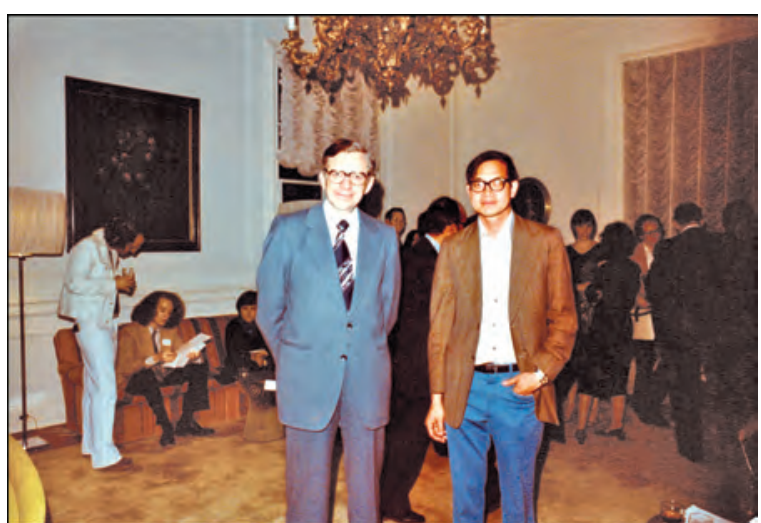

Grauert and Siu at Princeton, 1979.

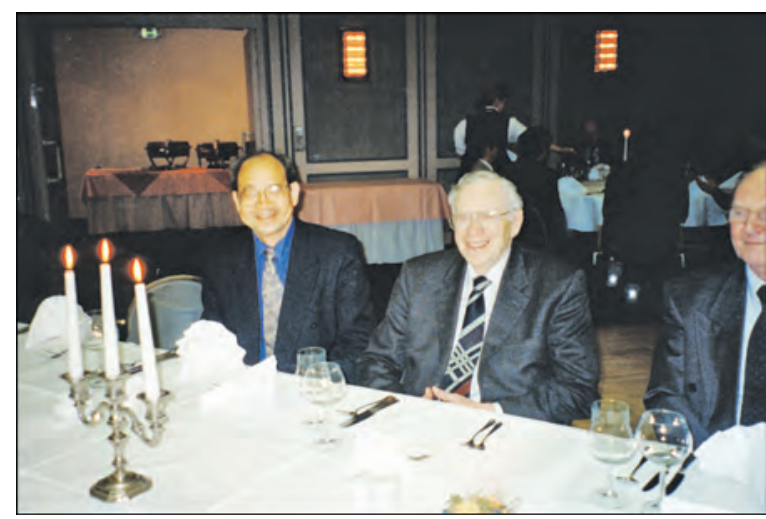

Grauert and Siu, 2000. Seventieth birthday in Göttingen.
As an offshoot of his solution of the Levi problem by his bumping technique, Grauert observed that the total space of a negative line bundle over a compact complex space is strictly pseudoconvex. With this observation Grauert established a link between his bumping technique of constructing holomorphic functions and the vanishing and embedding theorems of Kodaira in algebraic geometry, thereby extending the latter to the setting of singular spaces. Such a connection also led to the development of his important theory of modifications and exceptional analytic sets.

Later in 1962 in joint papers with Aldo Andreotti, Grauert generalized his bumping technique to yield finiteness theorems for $p$-pseudoconvex and $q$ pseudoconcave spaces and coherent sheaves with cohomological codimension conditions and to construct compactifications of pseudoconcave ends.

The Oka principle is the general philosophical framework concerning the equivalence between topological and holomorphic objects over Stein spaces. Grauert developed Runge approximation techniques for functions with values in holomorphic fiber bundles to prove the equivalence between topological and holomorphic fiber bundles over Stein spaces. Grauert's approximation technique was later applied by others to yield important results on embedding and immersion dimensions and the number of defining equations for submanifolds of Stein manifolds. To date, Grauert's equivalence and results from generalizations of his approximation techniques have remained the best rigorously proved cases of the Oka principle.

The direct image theorem of Grauert gives the coherence of the direct images of coherent sheaves under proper holomorphic maps. Grauert proved it by introducing a smoothing method to relate the norm estimates of cochains in two different Stein coverings for the same cohomology class in order to guarantee the convergence of power series whose coefficients are inductively defined elements of Fréchet spaces. Grauert's smoothing method resembles the smoothing process used in the Nash-Moser implicit function theorem in Nash's 1956 Annals of Mathematics paper and Moser's 1966 Annali della Scuola Normale Superiore di Pisa paper. Although later in the early 1970s ForsterKnorr and Kiehl-Verdier gave simpler proofs of Grauert's direct image theorem, yet even in the simpler proofs there is still some analogue of Grauert's key smoothing process.

While at Göttingen, Grauert made Göttingen the mecca of several complex variables. The golden foundational period of the modern theory of several complex variables started with the work of the schools of Behnke, Oka, Cartan, Serre, Stein, Remmert, Andreotti, and many others and culminated in the phenomenal contributions of Grauert in the late 1950s and early 1960s. In Göttingen, between 1960 and 1996 Grauert produced forty Ph.D. students. Through visitor programs in Göttingen and international conferences Grauert mentored an entire generation of young mathematicians working on the theory of several complex variables. He set the research agenda in the field by trailblazing in a broad range of new directions, such as non-Archimedean function theory, integral formulas, deformation of singularities, low-rank vector bundles, and many others.

The first time I met Professor Grauert in person was in the several complex variables conference in Maryland in 1969. Since then he has invited me to visit Göttingen a number of times. Grauert was a man of very few words. Yet his concise, to-the-point professional comments carried great impact. Grauert was very kind, affable, and generous. Whenever he was in the company of young mathematicians, he always inspirationally shared without any hesitation his mathematical ideas with anybody within earshot. Jointly with Remmert and 


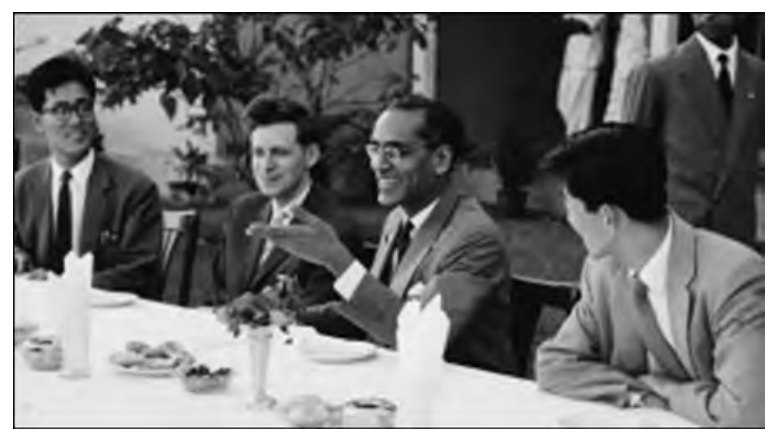

Kawai, Grauert, Chandrasekharan, Kuranishi, 1960. International Colloquium on Function Theory, Tata Institute in Bombay.

Stein, for many years Grauert organized workshops in several complex variables in Oberwolfach. I still recall with nostalgic fondness those casual after-dinner conversations in the lounge area outside the library. While sipping German white wine priced at ten marks a bottle, I and other junior mathematicians sat in a circle around Grauert and other senior mathematicians, attentively taking in Grauert's remarks about his mathematical ideas and viewpoints.

During all my visits to Göttingen, I benefited immensely from the many stimulating mathematical discussions in his office and in walks with him around Göttingen. I especially enjoyed my visit in 1993, when Grauert was compiling his own selected papers and invited me to Göttingen to keep him up to date concerning the impact of his work on the field. We conversed daily about his panoramic views of mathematics in general and about his own work. His recounting of the circumstances and his thought processes which led to his results were to me a real eye-opener and a sumptuous intellectual feast.

My conversations with Grauert rarely strayed far from the subject of mathematics. In one of my visits to Göttingen, he took me to the Gauss tower near Göttingen and we had lunch in the restaurant there, surrounded by brass instruments devised and used by Gauss. He went into a long discourse about Gauss and, in particular, about how a committee, which was charged with the responsibility of singling out one contribution of Gauss to put on the ten mark bill honoring Gauss, eventually came up with the choice of the Gaussian distribution in probability. He then told me that when he was a student he was very interested in physics. He began to focus his attention on mathematics only after he accidentally broke a galvanometer in a physics laboratory and was shown his way out of the laboratory. It was the good fortune of mathematics that the accidental breaking of a lowly galvanometer at that time led later to a complete change of the landscape of the modern theory of several complex variables.

Grauert was an intellectual giant. Those of us who had the good fortune of coming under his tutelage are not only awed by his brilliant mind as a mathematician but inspired, too, by his personal qualities. He was truly a shining example of integrity and magnanimity.

\section{Takeo Ohsawa}

\section{Thesis, Smiles, and Beyond}

It was in January 1980 when I went to Göttingen to see Grauert. I really wanted to meet him in person because I was strongly impressed by his masterpieces. In Grauert's papers I witnessed how excellent ideas work, feeling the strength of simplicity of arguments. Not to mention the great achievements, including the solution of the Levi problem on complex manifolds (see [G3]), I highly appreciated his thesis [G1] on the domains with complete Kähler metrics because it contained a result contrary to my naive guess.

In order to realize the desired trip from Kyoto to Göttingen, I had to write a letter to Grauert to get his approval for the application to the Alexander von Humboldt Foundation. In that letter I enclosed my master's thesis showing that a nonsingular rational curve embedded in a complex manifold admits a holomorphically convex neighborhood whenever the normal bundle is seminegative. (This was an answer to a problem which A. Fujiki had given to me after I finished reading [G5].) To my great pleasure I received a positive answer. In the letter Grauert simply and strongly wrote, "I will be glad if you can come." As a result, it was possible for me to spend sixteen months in Göttingen. I happily remember that Grauert welcomed me into his office with a distinctive smile and a few days later introduced me as a student of Shigeo Nakano (1923-1997) to the members of the seminar including Michael Schneider (1947-1997). It was good for me that everybody there seemed to know the Akizuki-Nakano vanishing theorem.

Among my pleasant experiences in Göttingen following this good start, let me recall an incident by which Grauert influenced my direction of research. Shortly before the summer break, Yum-Tong Siu visited us for several weeks. On Sundays Grauert took Siu and me in his Mercedes to the suburbs. Around noon on a sunny day, we saw the mountain on which Gauss measured relatively big triangles. The next day Siu gave a seminar talk. It was on the compactification of complete Kähler manifolds

Takeo Ohsawa is professor of mathematics at Nagoya University, Japan. His email address is ohsawa@math . nagoya-u . ac . jp. 
(see [SY]). I remember that he used the word "spike" to describe the shape of the ends of manifolds. At the end of the talk, Grauert made a remark that it was still an open problem whether or not complete Kähler domains with $\mathrm{C}^{1}$-smooth boundary are locally pseudoconvex. I was happy to hear this because I had already submitted a paper solving it affirmatively (see [O]). Why not capture this wonderful chance! So, after hearing Siu say that all that he knew was Grauert's affirmative solution [G1] for domains with real analytic boundary, I proposed to explain why $\mathrm{C}^{1}$-smoothness suffices. Allowed by Grauert, I did it in ten or fifteen minutes because I could skip some part of the $L^{2}$-theory. Grauert seemed to be convinced and applauded me by knocking on the table, with a contented smile. All of this happened in a lecture room where very large portraits of Klein and Hilbert were hung.

Encouraged by this experience, I tried to optimize the method of extending holomorphic functions with $L^{2}$-growth conditions and eventually arrived at a result [OT] which recently turned out to be useful for many purposes. In the selected papers of Grauert [G9] with his own commentary, $[\mathrm{O}]$ is referred to but [OT] is not. So I wish to supplement his commentary on [G1] by adding a remark: The idea of understanding the role of pseudoconvexity via geometry of Kähler manifolds yielded an effective extension technique in several complex variables.

\section{Jean-Pierre Demailly}

\section{Hans Grauert and the Foundation of Modern Complex Analysis}

Hans Grauert was born in 1930 in Haren, a town in Niedersachsen, Germany. He passed away on September 4, 2011, leaving the mathematical world with an extraordinary legacy in complex analysis and analytic geometry. I would like to share here a few recollections of my encounters with him during the last decades and some connections of his work with my own research. I first met Hans Grauert at the end of the 1970s on the occasion of a conference in Paris and still remember very well a discussion we had then. I was a young student at that time, and on the detour of a naive question I had raised, he had to explain the concept of a meromorphic map $X \rightarrow Y$ between complex manifolds.... Of course, I had already been somewhat acquainted with Grauert's major contributions to the theory of analytic spaces [GR1], [G5] and the Levi problem [G1], [G3] - and as a consequence was very impressed to exchange a few words with him.

Jean-Pierre Demailly is professor of mathematics at the Institut Fourier, Laboratoire de Mathématiques, France. His email address is Jean-Pierre.Demai11y@ujf-grenoble.fr.

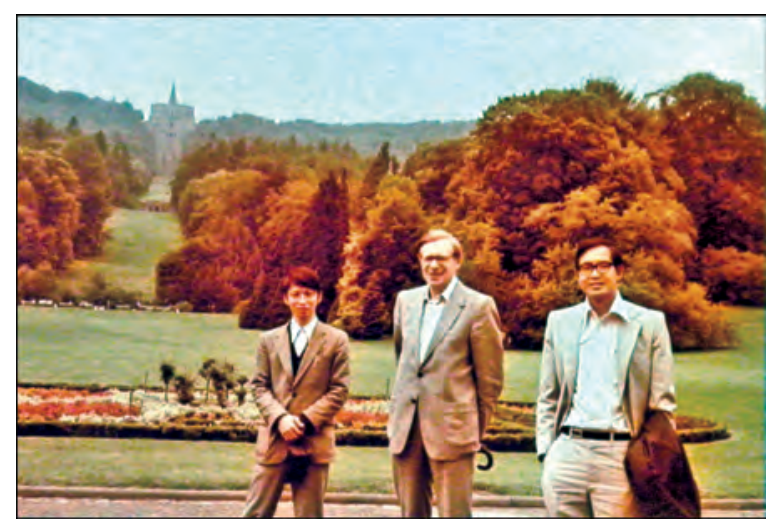

Ohsawa, Grauert, and Siu at Kassel-Wilhelmshöhe, 1980.

In 1986, Hans Grauert had heard more of my work, and he invited me to spend a couple of weeks in Göttingen; my stay actually took place in November 1986. At that time, Grauert was interested in the study of Kobayashi hyperbolicity, especially in view of his recently published paper "Hyperbolicity of the complement of plane curves" [GP], in collaboration with his daughter Ulrike Grauert-Peternell. He raised on the occasion a number of tough questions about hyperbolicity, and at that time I could not even think of any possible attempt to investigate them. Anyway, the discussion was to have a profound influence on my thinking years later-I will give a few more details on this below. It was also around that period that Grauert had received a private copy of Grothendieck's writing "Récoltes et semailles" [Gro2], a very personal account of an important period of Grothendieck's mathematical life. Grauert had already been in close contact with Grothendieck at the end of the 1950s, culminating with their work on the direct image theorem for coherent sheaves (in the algebraic and analytic settings, [G3] and [G4], respectively). Although he was obviously not at all targeted, I remember that Grauert was a bit upset about some of the controversial sentences contained in Grothendieck's testimony....

Coming back to Kobayashi hyperbolicity theory, Grauert introduced in [G8] the important concept of a jet metric, following previous work by Green and Griffiths [GG] on jet differentials. If $X$ is a projective nonsingular variety, let us consider $J_{k} X$ to be the bundle of $k$-jets of holomorphic curves $f:(\mathbb{C}, 0) \rightarrow X$, together with the $\mathbb{C}^{*}$-action $(\lambda \cdot f)(t)=f(\lambda t)$ obtained by reparameterizing the curve with a linear change of parameter. One can consider the projectivized jet bundle $X_{k}:=J_{k} X / \mathbb{C}^{*}$ whose fibers are weighted projective spaces and the corresponding $\mathcal{O}_{X_{k}}(1)$ tautological sheaf. A $k$-jet metric is then just a hermitian metric on that sheaf; in other words, this is a nonnegative function 


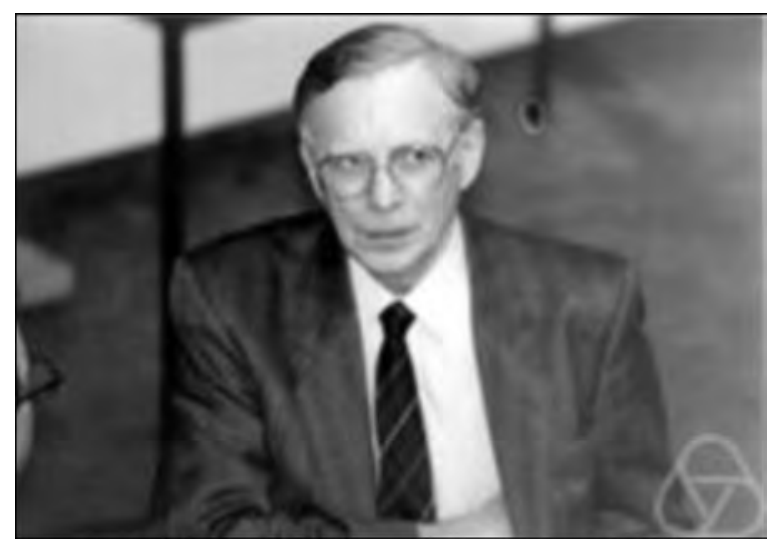

Hans Grauert, 1990.

$\rho: J_{k} X \rightarrow \mathbb{R}^{+}$such that $\rho(\lambda \cdot f)=|\lambda| \rho(f)$, in Grauert's own notation. By looking at all holomorphic curves $f: \Delta \rightarrow X$ on the unit disk possessing a prescribed $k$-jet at 0 up to the $\mathbb{C}^{*}$-action and trying to maximize the multiple, one defines in a canonical way a $k$-jet metric $\rho_{k, \text { can }}$ which is just the $k$-jet analogue of the Kobayashi infinitesimal metric in the case $k=1$. Grauert realized that, under a suitable negativity assumption for the curvature of the $k$-jet metric $\rho$, the Ahlfors-Schwarz lemma would imply the Kobayashi hyperbolicity of $X$; he then asked what type of curvature estimates the $k$-jet metric $\rho_{k, \text { can }}$ should satisfy. This question, which was further explained to me by Grauert's younger collaborators Gerd Dethloff and Siegmund Kosarew, is still unsolved at present. In fact, it is convenient to introduce a variant of these jet bundles (replacing the $\mathbb{C}^{*}$-action by the group $\mathbb{C}_{k}$ of $k$-jets of biholomorphisms $\varphi:(\mathbb{C}, 0) \rightarrow(\mathbb{C}, 0)$; cf. [D2]), and then the conjecture is expressed by saying that $X$ is Kobayashi hyperbolic if and only if there exists a $k$-jet metric with strictly negative curvature (in a suitable sense), with poles contained in the set of $k$-jets that are singular at the origin, for all $k \geq k_{0}$ large enough. In this statement, it can be shown, starting with hyperbolic complex surfaces, that $k_{0}$ may have to be taken arbitrarily large. In the positive direction, it can be derived from a recent result of [D3] that a projective manifold of general type always possesses a negatively curved $k$-jet metric for $k$ large if one forgets about the demands on the set of poles, thus proving only some sort of weak generic hyperbolicity of $X$. This is done by studying the cohomology of the bundles of jet differentials and inferring from that that every entire holomorphic curve $f: \mathbb{C} \rightarrow X$ has to satisfy global algebraic differential equations $P\left(f ; f^{\prime}, \ldots, f^{(k)}\right)=0$; in fact $f$ has to satisfy a large number of them when $k$ increases.

Another foundational result is the GrauertRiemenschneider theorem [GRi] : if $X$ is a projective or Moishezon manifold, then $H^{q}\left(X, K_{X} \otimes L\right)=0$ for every $q \geq 1$ and every semiample line bundle $L \rightarrow X$ of maximal Kodaira dimension. The result is often used in its relative form, stating that if $\mu: X \rightarrow Y$ is a projective birational morphism over some base $Y$, then all higher direct images $R^{q} \mu_{*} \mathcal{O}\left(K_{X}\right)$ vanish $(q \geq 1)$. In the same paper, Grauert and Riemenschneider conjectured that a compact analytic space is Moishezon if and only if it carries an almost positive coherent sheaf of rank 1; cf. also [R1]. Pursuing these ideas, Oswald Riemenschneider solved the Kähler case in [R2] by showing that a compact Kähler manifold carrying a line bundle whose curvature is semipositive and strictly positive at one point is actually Moishezon. The general case (removing the Kähler assumption) was finally settled by Yum-Tong Siu in [S1], using very clever bounds on Čech cohomology classes and their harmonic counterparts. These results served as the main motivation and as a strong guide for the discovery of holomorphic Morse inequalities in [D1] (probably the reason for Grauert's interest in my work in 1986 and, incidentally, also one of the main ingredients for the above-mentioned result of [D3]). These inequalities can be stated as follows: for every compact complex manifold $X$ and every holomorphic line bundle $L$, we have as $k \rightarrow+\infty$ the asymptotic estimate of cohomology groups

(*) $\begin{aligned} & \frac{n !}{k^{n}} \sum_{j=0}^{q}(-1)^{q-j} h^{j}\left(X, L^{\otimes k}\right) \\ & \quad \leq \int_{X(u, \leq q)}(-1)^{q} u^{n}+o(1), \quad n=\operatorname{dim}_{\mathbb{C}} X,\end{aligned}$

where $u$ is a smooth closed $(1,1)$-form in $c_{1}(L)$ and $X(u, \leq q)$ is the open set of points $x \in X$ where $u(x)$ is nondegenerate with at most $q$ negative eigenvalues. Assuming $\int_{X(u, \leq 1)} u^{n}>0$, the estimate implies that $L^{\otimes k}$ has many sections, hence that $L$ is big and that the base manifold $X$ is Moishezon. Using a slight improvement due to L. Bonavero [B] ("singular holomorphic Morse inequalities"), one concludes that a compact complex manifold is Moishezon if and only if it carries a holomorphic line bundle possessing a singular hermitian metric $h$ with analytic singularities such that the curvature current $u=\Theta_{L, h} \in c_{1}(L)$ satisfies $\int_{X(u, \leq 1) \backslash Z} u^{n}>0$, on the complement of the set of poles $Z \subset X$. This characterization strengthens Siu's solution of the Grauert-Riemenschneider conjecture.

Among Grauert's other fundamental contributions, the Andreotti-Grauert theorem [AG] stands out as one of the most important finiteness theorems of analytic geometry. Let us recall that $a$ complex $n$-dimensional complex manifold $X$ is said to be q-convex (resp. q-complete) if $X$ possesses a smooth exhaustion function $\varphi$ such that the Levi form $i \partial \bar{\partial} \varphi$ has at least $n-q+1$ strictly positive 
eigenvalues on the complement $X \backslash K$ of a compact set (resp. on $X$ itself); an appropriate definition can also be given for arbitrary complex spaces. Along with many other results, [AG] proves that the cohomology groups $H^{j}(X, \mathcal{F})$ of a coherent analytic sheaf $\mathcal{F}$ on $X$ are finite dimensional (resp. vanish) if $X$ is $q$-convex (resp. $q$-complete) and $j \geq q$. This finiteness statement implies a very interesting corollary also in the compact case. Let $(L, h)$ be a hermitian line bundle on a compact complex manifold $X$ such that the curvature form $\Theta_{L, h}=-\frac{i}{2 \pi} \partial \bar{\partial} \log h$ has at least $n-q+1$ positive eigenvalues at every point. Then the total space of the dual line bundle $L^{*}$ is $q$-convex, and one can easily derive from this that $H^{j}\left(X, L^{\otimes k}\right)=0$ for $j \geq q$ and $k$ large enough. The holomorphic Morse inequalities would yield here the related (but somewhat less precise) result that $h^{j}\left(X, L^{\otimes k}\right)=o\left(k^{n}\right)$ for $j \geq q$, whenever $\Theta_{L, h}$ has at least $n-q+1$ semipositive eigenvalues at every point. An important unsolved question is whether a converse of the Andreotti-Grauert theorem holds true: assuming that $X$ (resp. $L$ ) is cohomologically q-convex, in the sense that the relevant cohomology groups $H^{j}(X, \mathcal{F})$ are finite dimensional (resp. vanish, resp. $\left.H^{j}\left(X, L^{\otimes k}\right)=0, k \gg 0\right)$ for $j \geq q$, does it follow that $X$ is q-convex (resp. q-complete); resp. does it follow that $L$ possesses a hermitian metric $h$ with the required signature? The latter question has been analyzed in more depth in [DPS]. In general, the answer is unknown except in the strictly pseudoconvex case $(q=1)$. Very recently, a partial converse was settled for line bundles $L$ over compact complex surfaces $\left(n=\operatorname{dim}_{\mathbb{C}} X=2\right)$, in the form of an asymptotic Morse equality

$$
\begin{aligned}
&(* *) \quad \limsup _{k \rightarrow+\infty} \frac{n !}{k^{n}} \sum_{j=0}^{q}(-1)^{q-j} h^{j}\left(X, L^{\otimes k}\right) \\
& \quad=\inf _{u \in c_{1}(L)} \int_{X(u, \leq q)}(-1)^{q} u^{n}, \quad 0 \leq q \leq n
\end{aligned}
$$

(cf. [D4]), the case of complex curves $(n=1)$ also being easy to check. It would be interesting to know whether such a result holds true when $n \geq 3$.

Hans Grauert has contributed many more fundamental results than those briefly discussed here. He can be considered as one of the founders of modern analytic geometry, and his achievements will certainly provide very strong guidelines for future research in the field. I have been deeply influenced by the research directions he initiated and also strongly encouraged by the role he played in the recognition of my work as a member of the Mathematisches Institut and of the Akademie der Wissenschaften zu Göttingen.

\section{Daniel Barlet}

\section{In Memory of Hans Grauert}

Throughout the years I have been impressed by the dynamism and quality of the German school of several complex variables under the influence of H. Grauert and R. Remmert. As a permanent invited guest to most conferences organized by this group, in Oberwolfach or elsewhere, for almost forty years, I have had the privilege of following and admiring the mathematical guidance of Hans Grauert in this school. Furthermore, it is evident that he also had a strong influence on our group in Nancy (among many others).

It was not so easy having mathematical discussions with Hans Grauert, and it was often difficult to follow him when he explained his works during these conferences. I have the feeling that his way of thinking about mathematics was not the usual way of ordinary people like me. I confess that it was more enlightening for me to think about his beautiful results and methods by myself than trying to follow him during these talks, and, in some sense I think it was the same for him in that he preferred to follow results of other mathematicians in his own way. But it is clear that from his special point of view and way of looking at mathematics, he was able to see and to understand many beautiful results that ordinary people cannot see. This insight would have been useless if he had not also had great strength for solving the technical problems arising along the way. But maybe I'm just giving a description of what an exceptional mathematician is.

\section{Günther Trautmann}

\section{On Grauert's Early Days in Göttingen}

When I entered Göttingen University in 1960 to study mathematics, I was supposed, as was the standard of this time, to follow a course in "Infinitesimalrechnung" and "Analytische Geometrie". The first was offered by the thirty-year-old Hans Grauert, the second by the then sixty-seven-yearold Kurt Reidemeister. While the first one was extremely formal for us, in the second we experienced an explanation of ideas seemingly without convergence to concrete results, a tremendous but fruitful contrast in teaching. Beforehand, older students had frightened me by saying that the lecture

Daniel Barlet is professor of mathematics at the Institut de Mathématique Élie Cartan, Université de Lorraine, France. His email address is Danie1. Bar1et@univ-1orraine.fr.

Günther Trautmann is professor of mathematics at Technische Universität Kaiserslautern, Fachbereich Mathematik, Germany. His email address is trm@ mathematik.uni-k1.de. 
of Grauert would formally follow the pattern "definition, proposition, proof" without comment. This was true, but, to the contrary, many of my fellow students and I appreciated this style because we received the complete formalism and a secure and steady way to higher results and thereby learned what it meant to be precise in doing our own work. What the warning of the older students did not contain was that by the careful organization of his lectures Grauert approached the contents in a very efficient and short way, e.g., Stokes's theorem for differential forms at the end of the first year. Later I learned that he was much less formal in his mathematical thinking.

In this first year I was not aware that the young Hans Grauert had already published several of the most important papers in complex analysis at that time, e.g., the work on the Oka principle for holomorphic vector bundles [G2], the solution of the Levi problem, and the famous theorem of the coherence of direct images under proper mappings [G4]. Here I cannot refrain from making a few comments on some of these papers that had an enormous impact in algebraic geometry and were guidelines for me.

\section{Some Mathematical Landmarks of Grauert}

The paper [G4] is certainly his most famous and his most technically complicated. It was called "Blaue Arbeit" because of the cover of the IHES offprint and because some of its proofs seemed mysterious to us. In fact, it took another decade before improved versions of the proof and generalizations appeared in common papers of Knut Knorr and Otto Forster and of Reinhardt Kiehl and Jean-Louis Verdier. Previous theorems on special cases for proper holomorphic mappings then appeared as corollaries and, as Alan Huckleberry puts it in $[\mathrm{H}]$, "one cannot think of working in global complex geometry without the availability of this result." The typical method in this paper is the construction of shrinking coverings together with estimates of operators between spaces of Čech cocycles which occurred in the unwrapping of the problem and which were linked in a complicated induction process. Such estimates were also used in the important paper [AG] with Aldo Andreotti on the finiteness of cohomology groups for concave and convex complex spaces. My own work, some of it with coauthors, on the extension of coherent analytic sheaves was inspired by this paper, as was the later work of Yum-Tong Siu on the coherence of direct images under concave-convex mappings.

In [G5] Hans Grauert found the remarkable link between pseudoconvexity and contractibility of divisors. His criterion for contractibility, expressed in terms of negative definite matrices, has become one of the important theorems

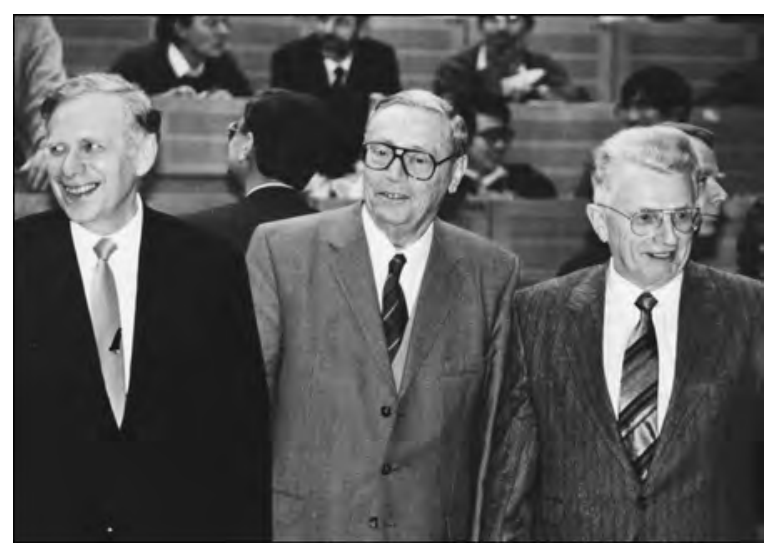

Grauert, Stein, and Remmert, 1990.

in the theory of complex surfaces. Another landmark in complex algebraic geometry was the Grauert-Riemenschneider vanishing theorem, [GRi], which had a lasting influence on further works on vanishing results.

In a later period Grauert's papers [G6] and [G7] in deformation theory became as important and pioneering as his previous ones, proving the existence of versal deformations. In [G6] he developed his method of reduction of analytic ideals in order to prove convergence of formal deformations, a method used later on in many other papers on deformations of holomorphic vector bundles, coherent analytic sheaves, and more general analytic objects. His method even works nowadays in computer algebra systems in order to calculate deformations. The existence of versal deformations for the global case of compact complex spaces was proved in [G7] in competition with similar efforts of Adrian Douady.

In the mid-1970s the question of the existence of small rank indecomposable vector bundles in projective $n$-space arose, which is considered as one of the most interesting questions in projective algebraic geometry. The Grauert-Mülich theorem on the behavior of jumping lines of stable vector bundles is one of the cornerstones for the classification of such bundles. Grauert was very interested in classifying algebraic vector bundles on higherdimensional projective spaces. Many questions, even for rank two vector bundles, remain open today. I remember one of the last conversations with Grauert about that problem, where we were betting, for lack of a well-founded conjecture, whether and which vector bundles of low rank would occur or not.

\section{The Göttingen Seminar}

Many of his former students may remember Grauert's lecture on algebraic topology in his third 


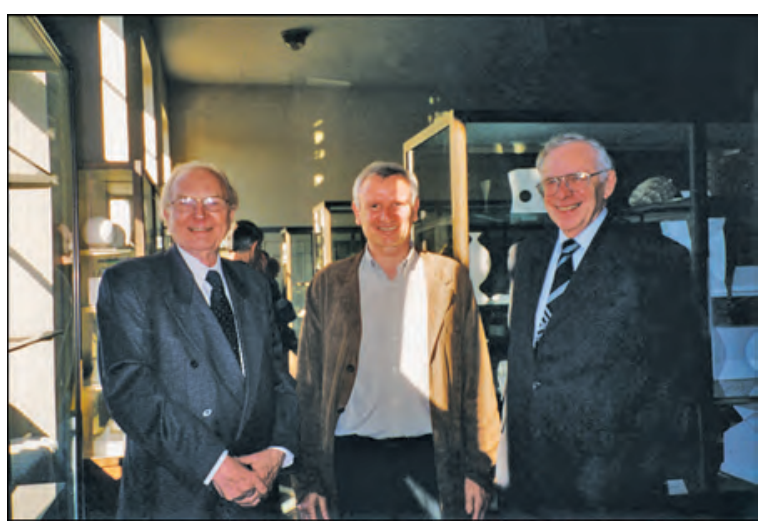

Grauert's seventieth birthday celebration in Göttingen. Hans-Heinrich Voigt, president of the Göttingen Academy of Sciences, Trautmann, and Grauert.

year at Göttingen, which at this time was revolutionary at Göttingen and marked the beginning of a new mathematical branch of the Göttingen institute, the branch of complex analysis with its "Oberseminar". It was my good fortune to have been in this seminar at this time. In this seminar we all learned about and gave talks on sheaf theory and pseudoconvexity. Shortly thereafter the group doubled when Reinhold Remmert joined Göttingen. This seminar can be considered the Göttingen continuation of the "Sturm und Drang" period in complex analysis described by Remmert in [R]. The seminar took place at 5 p.m. every Monday. Each Monday at 3 p.m. Grauert was present in his office for "Sprechstunde", where we could ask him about special problems concerning our projects for diploma and doctoral theses or talks. I remember one occasion when I told him about texts and papers I was reading or planned on reading. He said, "Lesen Sie nicht so viel, vieles Lesen macht dumm" (don't read so much; much reading makes stupid). It was always his intention to make us think on our own and push things through. A mathematician should develop his own imagination on the material he is working on. Another time when I could not settle a partial problem for my doctoral thesis he was sitting at his desk for about five (for me endless) minutes without saying a word, thinking of a solution and then told me to look at a Koszul complex, and indeed this was the way out. This mirrors the way he promoted and encouraged his students.

In the stimulating atmosphere of Göttingen's complex analysis we also appreciated the lectures of Reinhold Remmert on commutative algebra and the precise lectures of Emeritus Carl Ludwig Siegel on automorphic and modular functions. This lecture was held parallel to Grauert's lecture on complex analysis and was later published in the three volumes [S]. The contrast between a classical viewpoint in mathematics and the modern language of ringed spaces and functors became apparent in the controversies between Siegel and Grauert which enriched our studies.

At this time Grauert and Remmert had already written the larger part of the manuscripts of the books Stein Spaces and Coherent Analytic Sheaves which we used for the talks. There were many distinguished guests, among them Heisuke Hironaka talking about his work on desingularization before it was completed. So the time of the first half of the 1960s was the beginning of a very fruitful period of complex analysis in Göttingen. Many of Grauert's doctoral students later became university professors. The continuously successful complex analysis group of Hans Grauert and his later colleagues Michael Schneider and Hubert Flenner led to the establishment of a DFG- "Sonderforschungsbereich" for the Mathematical Institute in Göttingen in the 1980s. Its activities had a large and long-lasting impact in complex and algebraic geometry.

\section{Ingo Lieb}

\section{Hans Grauert: Teacher Extraordinary}

Hans Grauert is rightly considered as one of the leading mathematicians of the last half-century. His research achievements have made him known worldwide and have initiated substantial further developments in complex analysis and algebraic geometry. In the following lines I will describe a different, less spectacular, part of his work: his teaching. Through his students this has led to further significant contributions to mathematics. Let me first go back to the time of my studies at Göttingen and look at Grauert...

\section{Through the Eyes of a Student}

The schedule of my second year of mathematics at Göttingen University (1959) contained the theory of functions of a complex variable. A young professor - just some eight years older than his studentswho had come from Münster as the successor to Carl Ludwig Siegel, took charge of this course: Hans Grauert. I vividly remember the lecture and the lecturer: a slender, tall, young, and even younger looking, lecturer, giving the impression of great personal modesty, even shyness, very few motivating remarks, but absolute completeness of the mathematical arguments, never skipping any details; rarely had I understood a mathematical

Ingo Lieb is professor of mathematics at MathematischNaturwissenschaftliche Fakultät, Friedrich-Wilhelms Universität Bonn, Germany. His email address is $11 \mathrm{ieb@math.}$ uni-bonn. de. 
theory so directly or had been so impressed by its beauty. These characteristic features of Grauert's teaching style did not change very much in the course of the years. True, he exuded quite naturally more self-assurance in later years; moreover, he usually gave more motivating hints in his later lectures, usually very profound remarks, but in all his lectures he insisted on logical precision and complete arguments (the phrases trivial or as can be easily seen never came up in his lectures), and he always stepped behind the mathematical content, trying to make mathematics speak for itself. This is all the more remarkable as there would have been many instances where he might have quite rightly mentioned his own work, but I saw this only much later. (See "With the Eyes of a Coworker.")

\section{The Göttingen Oberseminar}

Starting around 1962, Grauert looked for students whom he could lead into mathematical research. To this end, he established his Oberseminar (graduate seminar), which he directed from 1962 until his retirement in 1995, at times jointly with Reinhold Remmert, Egbert Brieskorn, Michael Schneider, et al. Among the first members of this seminar were Günter Trautmann, Wolfgang Fischer, Ingo Lieb, Klas Diederich, Enrique Ramirez, Helmut Reckziegel, Rolf Richberg, Oswald Riemenschneider, Gudrun Kalmbach, all of whom became university teachers. Every single talk in that seminar was-without us clearly realizing it $-\mathrm{a}$ talk at the borderline of actual knowledge, and we, the students, were allowed to participate. The central mathematical topics in those days were the theory of Stein spaces, deformation theory, and analytic sheaf theory. We learned all these things from typed notes, which gave Grauert's and Remmert's own views of Stein theory and sheaf theory. These notes crystallized some years later in the famous three volumes of Grundlehren [GR2], [GR3], [GR4]. In addition, the seminar offered an opportunity to organize visiting lectures: H. Hironaka, F. Norguet, A. Douady, R. Wells, A. Andreotti, and many other leading researchers gave us talks.

Work in the Oberseminar has led altogether to forty-five Ph.D. theses supervised by Grauert, and in addition a considerable number of theses were supervised by his colleagues Remmert et al. The problems that Grauert suggested involved the whole area of complex analysis, from deformations of complex spaces to the geometry and analysis of domains of holomorphy and the boundary behavior of holomorphic maps. The overriding goal invariably aimed at substantial progress on major open areas of the field. He frequently gave indications of possible solutions generally in very concise, sometimes almost cryptic, formulation.

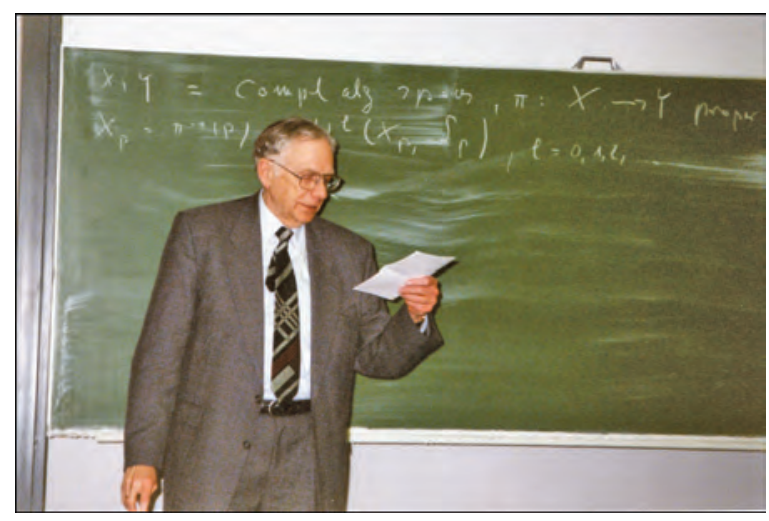

Beginning of a lecture. Grauert with his famous little piece of paper.

One was well advised to take each of his words seriously. His remarks were always founded on his previous deep preliminary reflections on the task in question. Sometimes one understood his hints only after one's own solution to the problem, just to see that his ideas had been right! Work on a problem posed by Grauert opened up, for a young, well-motivated mathematician, new fruitful fields of research, and no one was happier than Grauert when one of his students had successfully worked out his original ideas. Let me now see him...

\section{With the Eyes of a Coworker}

Hans Grauert loved to work jointly with his students. He wrote a joint paper with H. Reckziegel, when the latter was still an undergraduate [GRe]. More examples of this research in pairs stemming from Grauert's circle of ideas are joint papers with W. Fischer, I. Lieb, Michael Commichau, O. Riemenschneider [GFi], [GL], [CG], [GRi]. He particularly relied on his coauthors for his work on monographs and textbooks [GFL], [GFr]; Klaus Fritzsche, W. Fischer, I. Lieb, O. Riemenschneider [GR2] should be mentioned in this context. The books were usually based on preceding lectures, for which Grauert had detailed scripts written. This brings me back to my remarks in the first section: even standard chapters of the teaching material were freshly thought through and occasionally substantially transformed. For example, we find in his textbooks or lecture notes a new approach to Lebesgue integration (which easily carries over to functions with values in topological vector spaces but has been made accessible to first year undergraduates); a fairly general version of Stokes's theorem, probably valid even for semianalytic sets; a presentation of Maxwell's equations in the language of differential forms and currents clearly bringing to the foreground the invariance properties of the system (all this worked out for second year students!) [GFL]; an apparently 


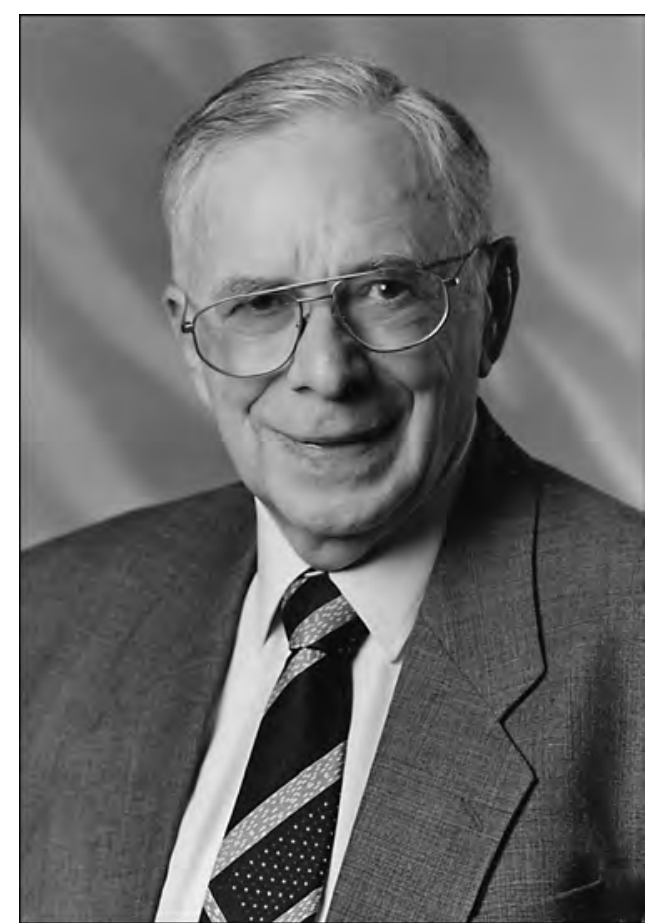

Hans Grauert in Bonn, 2000.

new construction of the completion of topological vector spaces [G10]; new proofs for the regularity of elliptic boundary value problems [G11]. In addition, the choice of topics, the choice between different options to build up and present a theory, shows Grauert's trademark: ex ungue leonem. The picture is completed by his monographs and textbooks on complex analysis [10], [13] in which many of his research results have found their place. So, Grauert would have had plenty of reason to mention himself in his lectures, but to my knowledge he never did it.

A last remark in this context: like most professors, Hans Grauert had to examine large numbers of students. In the German system this is mostly done in oral examinations. On these occasions he was a very mild and indulgent interview partner, as are, as I believe, most great scientists. As a recorder in these examinations, I have experienced this again and again.

Looking back...

\section{Through the Eyes of a Colleague}

The experiences with Hans Grauert that I have been describing here go back to the period between 1959 and 1971, when I was Grauert's student and coworker. This was a time when Grauert's amazing scientific productivity (starting around 1954) was at its peak. I did not notice this as a student, but later on as his colleague at Münster and Bonn I appreciated it and was deeply impressed. Research and teaching are in Grauert's work intimately intertwined. Einheit von Forschung und Lehre (unity of research and teaching) is Humboldt's university ideal, an ideal which seems quite unworldly in our time of impact factors, citation indexes, fights for and counting of grants, evaluations, professional formation, etc. Grauert realized this unity in his life: it is the same desire to know and the same urge for a profound understanding that characterizes his impressive research work and his lectures and textbooks.

\section{References}

[AG] A. ANDreotTi and H. GRAUERT, Théorèmes de finitude pour la cohomologie des espaces complexes, Bull. Soc. Math. France 90 (1962), 193-259.

[B] L. BONAVERo, Inégalités de Morse holomorphes singulières, C. R. Acad. Sci. Paris Sér. I Math. 317 (1993) 1163-1166.

[CG] M. Commichau and H. Grauert, Das formale Prinzip für kompakte komplexe Unterräume mit 1-positivem Normalebündel, Ann. Math. Stud. 100 (1981) 101126.

[D1] J.-P. Demailly, Champs magnétiques et inégalités de Morse pour la $d^{\prime \prime}$-cohomologie, Ann. Inst. Fourier (Grenoble) 35 (1985) 189-229.

[D2] Algebraic criteria for Kobayashi hyperbolic projective varieties and jet differentials, AMS Summer School on Algebraic Geometry, Santa Cruz, 1995 (J. Kollár and R. Lazarsfeld, eds.), Proc. Symposia in Pure Math., Amer. Math. Soc., Providence, RI, 1997, 285-360.

[D3] Holomorphic Morse inequalities and the Green-Griffiths-Lang Conjecture, Pure and Applied Math. Quarterly 7 (2011), 1165-1208.

[D4] A A converse to the Andreotti-Grauert theorem, Colloque "Analyse Complexe et Applications" en l'honneur de Nguyen Thanh Van (Octobre 2008), Annales Faculté Sciences Toulouse Math. 20 (2011), 123-135.

[DPS] J.-P. Demailly, Th. Peternell, and M. Schneider, Holomorphic line bundles with partially vanishing cohomology, Conf. in honor of F. Hirzebruch, Israel Mathematical Conference Proceedings, Vol. 9, 1996, 165-198.

[FG] K. FrITZSCHE and H. GRAUERT, From holomorphic functions to complex manifolds, Graduate Texts in Mathematics, 213, Springer 2002.

[G1] H. GRAUERT, Charakterisierung der holomorphvollständigen komplexe Räume, Math. Ann. 129 (1955), 233-259.

[G2] , Analytische Faserungen über holomorphvollständigen Räumen, Math. Ann. 135 (1957), 263-273.

[G3] On Levi's problem and the embedding of realanalytic manifolds, Ann. Math. 68 (1958), 460-472.

[G4] Ein Theorem der analytischen Garbentheorie und die Modulräume komplexer Strukturen, Publ. Math. I.H.E.S. 5 (1960), 233-292.

[G5] , Über Modifikationen und exzeptionelle analytische Mengen, Math. Ann. 146 (1962), 331-368.

[G6] _ Deformationen isolierter Singularitäten analytischer Mengen, Invent. Math. 15 (1972), 171-198. 
[G7]

— Der Satz von Kuranishi für kompakte komplexe Räume, Invent. Math. 25 (1974), 107-142.

[G8] , Jet Metriken und hyperbolische Geometrie, Math. Zeitschrift 200 (1989), 149-168.

[G9] _ Selected papers Vols. I, II, Springer-Verlag, 1994.

[G10] _ Funktionalanalysis in reeller und komplexer Analysis, Vorlesung, Göttingen 1979.

[G11]_ , Partielle Differentialgleichungen, Vorlesung, Göttingen, 1970.

[GFi] H. GRAUERT and W. FISCHER, Lokal-triviale Familien kompakter komplexer Mannigfaltigkeiten, Nachr. Akad. Wiss. Göttingen 6 (1965), 89-94.

[GFL] H. GRAUERT, W. FISCHER, and I. LIEB, Differential-und Integralrechnung, Vol. 3, Springer, 1966-1968.

[GFr] H. GRAUERT and K. FRITZSCHE, Einführung in die Funktionentheorie mehrerer Veränderlicher, Hochschultext, Springer, 1974.

[GL] H. GRAUERT and I. LIEB, Das Ramirezsche Integral und die Lösung der Gleichung $\bar{\partial} f=\alpha$ im Bereich der beschränkten Formen, Rice Univ. St. 56 (1970), 29-50.

[GRe] H. GRAUert and H. ReckZIEgel, Hermitische Metriken und normale Familien holomorpher Abbildungen, Math. Zeitschrift 89 (1965), 108-125.

[GR1] H. GRAUERT and R. REMMERT, Komplexe Räume, Math. Ann. 136 (1958), 245-318.

[GR2] _ Analytische Stellenalgebren, Grundlehren Math. Wiss., 176, Springer, 1971.

[GR3] _ Theorie der Steinschen Räume, Grundlehren der Mathematischen Wissenschaften, Vol. 227, Springer-Verlag, Berlin-New York, 1977.

[GR4] _ Coherent analytic sheaves, Grundlehren Math. Wiss., Vol. 265, Springer, 1984.

[GRi] H. GRAUERT and O. RIEMENSCHNEIDER, Verschwindungssätze für analytische Kohomologiegruppen auf komplexen Räumen, Invent. Math. 11 (1970), 263-292.

[GP] H. GRaUert and U. PETERnell, Hyperbolicity of the complement of plane curves, Manuscripta Math. 50 (1985), 429-441.

[GG] M. GREEN and P. GRIFFITHS, Two applications of algebraic geometry to entire holomorphic mappings, The Chern Symposium 1979, Proc. Internal. Sympos., Berkeley, CA, 1979, Springer-Verlag, New York, 1980, 41-74.

[Gro1] A. GRoTHENDIECK, The cohomology theory of abstract algebraic varieties, Proceedings of the Intern. Congress of Math., August 14-21, 1958, Edinburgh, Cambridge Univ. Press, 1960.

[Gro2] _, Récoltes et semailles, unpublished manuscript, available from http://www.math. jussieu.fr/ 1eila/grothendieckcircle/RetS. pdf.

[H] A. Huckleberry, Hans Grauert: Mathematiker Pur, Notices of the AMS 56, no. 1 (2009), 38-41.

[O] T. OHSAwA, On complete Kähler domains with $C^{1}$ boundary, Publ. RIMS 16 (1980), 929-940.

[OT] T. OHSAWA and K. TAKEGOSHI, On the extension of $\mathrm{L}^{2}$ holomorphic functions, Math. Z. (1987), 197-204.

[R] R. REMmERT, Complex analysis in "Sturm und Drang", The Mathematical Intelligencer 17, no. 2 (1995).

[R1] O. RIEMENSCHNEIDER, Characterizing Moishezon spaces by almost positive coherent analytic sheaves, Math. Zeitschrift 123 (1971), 263-284.
[R2] _ A generalization of Kodaira's embedding theorem, Math. Ann. 200 (1973), 99-102.

[S] C. L. SIEGEL, Topics in Complex Function Theory, Vols. I, II, III, Wiley-Interscience, 1969, 1971, 1973.

[S1] Y.-T. SIU, A vanishing theorem for semipositive line bundles over non-Kähler manifolds, Diff. Geom. 19 (1984), 431-452.

[S2] _ Some recent results in complex manifold theory related to vanishing theorems for the semipositive case, Proceedings of the Math. Arbeitstagung held in Bonn (June 1984), Max Planck Inst. für Math., Lecture Notes in Math., no. 1111, Springer-Verlag, 1985

[SY] Y.-T. SIU and S.-T. YAU, Compactification of negatively curved complete Kähler manifolds of finite volume, Seminar on Differential Geometry, Ann. of Math. Stud., Vol. 102, Princeton Univ. Press, Princeton, NJ, 1982, pp. 363-380. 